\title{
Detection and Analysis of Antimicrobial Resistance of Salmonella in Faeces of Foodborne Disease Patients
}

\author{
Liu Guihua, Huang Xin, Zhang Weiyu, Wang Yanqiu \\ Institute of Microbiology Department, Jilin Provincial Center for Disease Control and Prevention, Changchun, China \\ Email address: \\ liugh0523@sina.com (Liu Guihua), 46169267@qq.com (Huang Xin),81637146@qq.com (Zhang Weiyu), \\ 1416464882@qq.com (Wang Yanqiu)
}

\section{To cite this article:}

Liu Guihua, Huang Xin, Zhang Weiyu, Wang Yanqiu. Detection and Analysis of Antimicrobial Resistance of Salmonella in Faeces of Foodborne Disease Patients. Science Journal of Public Health. Vol. 8, No. 3, 2020, pp. 77-82. doi: 10.11648/j.sjph.20200803.13

Received: May 6, 2020; Accepted: May 22, 2020; Published: June 4, 2020

\begin{abstract}
Objective: To detect the drug resistance of Salmonella isolated from the faeces samples of patients with food-borne diseases, and to know the drug resistance status and development trend of food-borne pathogens in our province. Methods: Enrichment, color medium separation, API20E biochemistry and Thai serum Identification. The minimal inhibitory concentration (MIC) of Salmonella to 15 kinds of antibiotics were quantitatively determined by micro broth dilution method. Results: 123 food-borne Salmonella strains were resistant to ampicillin (AMP), NALIDIXIC acid (NAL), tetracycline (TET), trimethoprim/sulfamethoxazole (SXT), ampicillin / sulbactam (AMS), CEFAZOLIN (CFZ), chloramphenicol (CHL), CEFOTAXIME (CTX), ciprofloxacin (CIP), azithromycin (AZM), gentamicin (GEN), cefoxitin (CFX), ceftazidime (CAZ). The drug resistance rates were $87.80 \%, 84.6 \%, 69.1 \%, 50.4 \%, 39.0 \%, 34.1 \%, 26.0 \%, 26.0 \%, 26.0 \%, 24.3 \%, 19.5 \%, 2.4 \%$ and $0.8 \%$ respectively. IMP and ERY were both sensitive. Among them, 90 strains were resistant to more than 3 kinds of antibiotics, and the multidrug resistance rate was $73.2 \%$. MIC results ranged from $1 \mu \mathrm{g} / \mathrm{mL}$ to $>64 \mu \mathrm{g} / \mathrm{mL}$. The total strains were divided into 15 serotypings, including Salmonella Enteritidis (58.5\%), Salmonella Typhimurium (16.3\%), and Salmonella Stanley (4.9\%). The most common distribution of the 57 resistant strains were AMP-TET-NAL-and AMP-NAL-11 strains, followed by NAL-10 strains, AMP-AMS-TET-NAL-7 strains, and multi-resistant strains ranged from 3 to 11 antibiotics. Conclusion: The serotypings of Salmonella isolated from faeces samples of patients from food borne disease surveillance sites in hospitals in Jilin province were mainly S. Enteritis and S. Enterica, and the strains were resistant to multiple antibiotics. The surveillance of drug resistance of Salmonella in food-borne diseases should be strengthened to ensure food safety and human health.
\end{abstract}

Keywords: Foodborne Diseases, Broth Dilution, MIC, Salmonella, Results Analysis

\section{Introduction}

Salmonella was one of the most important zoonosis pathogens in public health, which could cause human gastroenteritis and food poisoning, and it was one of the main pathogens causing food poisoning in China [1-2]. According to the World Health Organization, the number of confirmed cases of Salmonella worldwide increased significantly since 1985, and increased fivefold in some European countries. Food poisoning caused by Salmonella often took the first place in inland areas of China. In 2016,123 strains of Salmonella isolated from faeces of patients with food-borne diseases in Jilin province were tested for drug resistance to 15 antibiotics [3-5].

\section{Materials and Methods}

\subsection{Source of Experimental Material}

\subsubsection{Source of Strains}

123 strains of Salmonella were isolated from food-borne disease surveillance sites in 22 hospitals throughout Jilin province.

\subsubsection{Culture Medium and Reagent Sources}

Columbia blood agar plate for culture medium and enrichment liquid and drug sensitivity test were purchased from Guangzhou Huankai Biotechnology Co. Ltd., API20E kit was produced by French Merieux Company, Salmonella serum was produced by Thailand and purchased from Beijing 
Luqiao Biotechnology Company, MIC kits for lyophilized bacteria quantitative drug susceptibility test were purchased from ThermoFisher Scientific Company.

\subsubsection{Quality Control Strain (E. Coli ATCC 25922) Source Ltd. \\ Purchased from Guangzhou Huankai Biotechnology Co.}

\subsection{Experimental Method}

\subsubsection{Method of Isolation and Identification}

The method of identification of the strains was carried out according to the method of National food-borne diseases manual [6].

\subsubsection{Drug Sensitivity Test}

The test was according to the standard for antimicrobial susceptibility test by disc diffusion of National committee for clinical laboratory/Clinical and Laboratory Standards Institute (NCCLS /CLSI2007)[7-10]. The broth dilution method was used. The quality control strain and the preparation strains were inoculated on Columbia blood agar plate respectively, pure cultured at $37^{\circ} \mathrm{C}$ for 24 hours. 3 to 5 pure new colonies were selected and suspended in a $10 \mathrm{~mL}$ normal saline dilution tube, and then the bacterial solution was adjusted to $0.5 \mathrm{MC}$ turbidimetric tube to make the bacterial content to $1 \times 10^{8} \mathrm{CFU} / \mathrm{mL}$. First, a micropipette was used in the Biosafety cabinet to suck up the turbid bacterial solution $10 \mu \mathrm{L}$ in a $12 \mathrm{~mL}$ broth tube, the mixture was mixed and slowly poured into a $\mathrm{V}$-shaped aseptic tank, and then added into 96-hole drug sensitive plate in turn with eight micropipettes (each hole $100 \mu \mathrm{l}$ ), covered with plastic wrap. The final inoculation concentration was about $1 \times 10^{5}$ to $2 \times 10^{5}$ (CFU/mL). $100 \mu \mathrm{L}$ bacterial suspension was added into the positive control hole. We should notice that aseptic operation of the whole process. The susceptibility test kits were cultured in $36 \pm 2{ }^{\circ} \mathrm{C}$, incubatored for 16 to 18 hours. Put a black plate under the Kit, observed with the naked eyes. Positive control pore turbidity showed that the bacteria grew well. If the bacteria in the pores were diffusely turbidity or the bottom of the hole had a round or reticular precipitation, represented bacteria grew in the pores. The minimum concentration of antibacterial drugs contained in the holes where bacteria grow was the minimum inhibitory concentration (MIC).

\section{Experimental Results}

\subsection{Results of Quality Control Report of Gram-Negative Drug Sensitive Plate}

According to Quality control report of Salmonella drug sensitivity test, the results of quality control strain test were within the scope of quality control [11]. See table 1.

Table 1. MIC tolerance range and MIC interpretation standard of quality-controlled strains $(\mu \mathrm{g} / \mathrm{mL})$.

\begin{tabular}{|c|c|c|c|c|}
\hline \multirow{2}{*}{ Name of antibiotic } & \multirow{2}{*}{ MIC range E. coli ATCC25922 } & \multicolumn{3}{|c|}{ MIC Standard of interpretation } \\
\hline & & Sensitivity & Mediation & Resistance \\
\hline AMP & $2 \sim 8$ & $\leq 8$ & 16 & $\geq 32$ \\
\hline AMS & $2 / 1 \sim 8 / 4$ & $\leq 8 / 4$ & $16 / 8$ & $\geq 32 / 16$ \\
\hline CFZ & $1 \sim 4$ & $\leq 2$ & 4 & $\geq 8$ \\
\hline CAZ & $0.06 \sim 0.50$ & $\leq 4$ & 8 & $\geq 16$ \\
\hline TET & $0.5 \sim 2$ & $\leq 4$ & 8 & $\geq 16$ \\
\hline ERY & - & $\leq 0.5$ & $1 \sim 4$ & $\geq 8$ \\
\hline NAL & $1 \sim 4$ & $\leq 16$ & - & $\geq 32$ \\
\hline CFX & $2 \sim 8$ & $\leq 8$ & 16 & $\geq 32$ \\
\hline CTX & $0.03 \sim 0.12$ & $\leq 1$ & 2 & $\geq 4$ \\
\hline GEN & $0.25 \sim 1$ & $\leq 4$ & 8 & $\geq 16$ \\
\hline SXT & $\leq 0.5 / 9.5$ & $\leq 2 / 38$ & - & $\geq 4 / 76$ \\
\hline $\mathrm{CHL}$ & $2 \sim 8$ & $\leq 8$ & 16 & $\geq 32$ \\
\hline IMP & $0.06 \sim 0.25$ & $\leq 1$ & 2 & $\geq 4$ \\
\hline
\end{tabular}

Note: No intermediate concentration was due to sensitivity and resistance of the two concentrations for the adjacent multiple concentration.

\subsection{Drug Sensitivity Test of the Experimental Strains}

The MIC results of 123 strains of Salmonella in broth dilution method were shown in table 2. All 123 food-borne Salmonella strains were resistant to AMP, NAL, TET, SXT, AMS, CFZ, CHL, CTX, CIP, AZM, GEN, CFX, CAZ in different degree. The rates of drug resistance and MIC ( $\mu \mathrm{g}$
$/ \mathrm{ml})$ results were: $87.8 \%(32 \sim \geq 64), 84.6 \%(32 \sim>64)$, 69.1\%(16 >32), 50.4\% (4 /76 >8 /152), 39.0\% (32 /16 $\geq 64 / 32), 34.1 \%(8 \sim>16), 26.0 \%(32 \sim>64), 26.0 \%$ $(4 \sim>8), 26.0 \%(1 \sim>32), 24.3 \%(32 \sim>64), 19.5 \%(16 \sim>32)$, $2.4 \%(32 \sim>64), 0.8 \%(\geq 16)$. IMP and ERY were both sensitive. See table 2.

Table 2. MIC results of 123 strains of Salmonella.

\begin{tabular}{lllllll}
\hline Antibiotics & $\mathbf{n}$ & Sensitivity & Mediation & Resistance & Drug resistance rate (\%) & MIC of resistant strain $(\boldsymbol{\mu g} / \mathbf{m l})$ \\
\hline AMP & 123 & 11 & 4 & 108 & 87.8. & $32 \sim \geq 64$ \\
CAZ & 123 & 117 & 5 & 1 & 0.8 & $\geq 16$ \\
AMS & 123 & 18 & 57 & 48 & 39.0 & $32 / 16 \sim \geq 64 / 32$ \\
\hline
\end{tabular}




\begin{tabular}{lllllll}
\hline Antibiotics & $\mathbf{n}$ & Sensitivity & Mediation & Resistance & Drug resistance rate (\%) & MIC of resistant strain $(\boldsymbol{\mu g} / \mathbf{m l})$ \\
\hline IMP & 123 & 123 & 0 & 0 & 0 & $*$ \\
TET & 123 & 33 & 5 & 85 & 69.1 & $16 \sim>32$ \\
NAL & 123 & 19 & 0 & 104 & 84.6 & $32 \sim>64$ \\
CFX & 123 & 112 & 8 & 3 & 2.4 & $32 \sim>64$ \\
CHL & 123 & 89 & 2 & 32 & 26.0 & $32 \sim>64$ \\
ERY & 123 & 123 & 0 & 0 & 0 & $*$ \\
CTX & 123 & 85 & 6 & 32 & 26.0 & $4 \sim>8$ \\
CFZ & 123 & 69 & 12 & 42 & 34.1 & $8 \sim>16$ \\
GEN & 123 & 92 & 7 & 24 & 19.5 & $16 \sim>32$ \\
SXT & 123 & 61 & 0 & 62 & 50.4 & $4 / 76 \sim>8 / 152$ \\
AZM & 123 & 93 & 0 & 30 & 24.3 & $32 \sim>64$ \\
CIP & 123 & 147 & 74 & 32 & 26.0 & $1 \sim>32$ \\
\hline
\end{tabular}

Note: * means no resistant strain.

\subsection{Serotyping Results of the Experimental Strains}

123 Salmonella strains were divided into 15 serotypings, the main serotypings were S. Enteritidis (58.5\%), S. Typhimurium (16.3\%), and S. Stanley (4.9\%). See table 3.

Table 3. Serotyping distribution of 123 strains of Salmonella.

\begin{tabular}{llll}
\hline Serotyping & n (\%) & Serotyping & n (\%) \\
\hline S. Enteritidis & $72(58.5)$ & S. Gdansk & $1(0.8)$ \\
S. Typhimurium & $20(16.3)$ & S. Litchfield & $1(0.8)$ \\
Stanley & $6(4.9)$ & S. Augustenbory & $1(0.8)$ \\
S. Abony & $3(2.4)$ & S. Agona & $1(0.8)$ \\
S. Typhi & $3(2.4)$ & S. Essen & $1(0.8)$ \\
S. Derby & $3(2.4)$ & S. Tarshyne & $1(0.8)$ \\
S. ParatyphiB & $2(1.6)$ & S. Bonn & $1(0.8)$ \\
S. Rissen & $1(0.8)$ & & \\
\hline
\end{tabular}

\subsection{Analysis of drug Resistance Profile of Experimental Strains}

57 drug resistance profile, the most common distribution were AMP-TET-NAL- and AMP -NAL-11 strains each, the second of all was AMP-AMS-TET-NAL-7 strains. Multi-resistant strains ranged from 3 to 11 kinds of antibiotics. 5 strains with 11 kinds of antibiotic resistance were divided into 3 kinds of antibiotic resistance profile; 7 strains with 10 kinds of antibiotic resistance were divided into 4 kinds of antibiotic resistance profile; 11 strains with 9 kinds of antibiotic resistance were divided into 7 kinds of antibiotic resistance profile; 2 strains with 8 kinds of antibiotic resistance were divided into two antibiotic resistance profile; 5 strains with 7 kinds of antibiotic resistance were divided into 3 antibiotic resistance profile; 5 strains with 11 kinds of antibiotic resistance were divided into 3 antibiotic resistance profile; 10 strains with 11 kinds of antibiotic resistance were divided into 5 antibiotic resistance profile; 7 strains of 6 kinds of antibiotic resistance were divided into 6 kinds of antibiotic resistance profile; 21 strains of 5 kinds of antibiotic resistance were divided into 14 kinds of antibiotic resistance profile; 15 strains of 4 kinds of antibiotic resistance were divided into 7 kinds of antibiotic resistance profile; 20 strains of 3 kinds of antibiotic resistance were divided into 6 kinds of antibiotic resistance profile; 10 strains of 1 kinds of antibiotic resistance were 1 kind of antibiotic resistance profile. See table 4.

Table 4. Drug resistance profile of 123 strains of Salmonella.

\begin{tabular}{|c|c|c|}
\hline Types of antibiotics & Drug resistance profile & Strains \\
\hline 11 & AMP-CAZ-AMS-TET-NAL-CTX-CFZ-GEN-SXT-AZM-CIP- & 1 \\
\hline 11 & AMP-AMS-TET-NAL-CHL-CTX-CFZ-GEN-SXT-AZM-CIP- & 3 \\
\hline 11 & AMP-TET-NAL-CFX-CHL-CTX-CFZ-GEN-SXT-AZM-CIP- & 1 \\
\hline 10 & AMP-TET-NAL-CHL-CTX-CFZ-GEN-SXT-AZM-CIP- & 3 \\
\hline 10 & AMP-AMS-TET-NAL-CHL-CTX-CFZ-SXT-AZM-CIP- & 2 \\
\hline 10 & AMP-AMS-TET-NAL-CTX-CFZ-GEN-SXT-AZM-CIP- & 1 \\
\hline 10 & AMP-AMS-TET-NAL-CHL-CTX-CFZ-GEN-SXT-AZM- & 1 \\
\hline 9 & AMP-AMS-TET-NAL-CHL-CTX-GEN-SXT-CIP- & 1 \\
\hline 9 & AMP-TET-NAL-CHL-CTX-CFZ-SXT-AZM-CIP- & 3 \\
\hline 9 & AMP-TET-NAL-CTX-CFZ-GEN-SXT-AZM-CIP- & 3 \\
\hline 9 & AMP-AMS-TET-NAL-CTX-CFZ-GEN-SXT-AZM- & 1 \\
\hline 9 & AMP-AMS-TET-NAL-CFX-CFZ-SXT-AZM-CIP- & 1 \\
\hline 9 & AMP-AMS-TET-NAL-CTX-CFZ-SXT-AZM-CIP- & 1 \\
\hline 9 & AMP-NAL-CHL-CTX-CFZ-GEN-SXT-AZM-CIP & 1 \\
\hline 8 & AMP-AMS-NAL-CTX-CFZ-SXT-AZM-CIP- & 1 \\
\hline 8 & AMP-AMS-TET-NAL-CHL-GEN-SXT-CIP- & 1 \\
\hline 7 & AMP-AMS-TET-NAL-CTX-CFZ-SXT- & 2 \\
\hline 7 & AMP-TET-NAL-CHL-GEN-SXT-CIP- & 1 \\
\hline 7 & AMP-TET-NAL-CHL-CTX-CFZ-SXT- & 1 \\
\hline 7 & AMP-TET-NAL-CFZ-SXT-AZM-CIP- & 4 \\
\hline 7 & AMP-NAL-CTX-CFZ-SXT-AZM-CIP---- & 2 \\
\hline 6 & AMP-AMS-TET-NAL-CHL-CFZ- & 1 \\
\hline 6 & AMP-AMS-TET-NAL-CFZ-SXT- & 1 \\
\hline
\end{tabular}




\begin{tabular}{|c|c|c|}
\hline Types of antibiotics & Drug resistance profile & Strains \\
\hline 6 & AMP-AMS-TET-NAL-CTX-CFZ- & 1 \\
\hline 6 & AMP-AMS-NAL-GEN-SXT-AZM- & 1 \\
\hline 6 & AMP-TET-NAL-CTX-CFZ-SXT- & 1 \\
\hline 6 & AMP-TET-NAL-CHL-SXT-CIP- & 2 \\
\hline 5 & AMP-AMS-TET-NAL-SXT- & 4 \\
\hline 5 & AMP-AMS-TET-NAL-AZM- & 1 \\
\hline 5 & AMP-AMS-TET-NAL-CFZ- & 1 \\
\hline 5 & AMP-AMS-CTX-CFZ-SXT- & 1 \\
\hline 5 & AMP-TET-NAL-CHL-SXT- & 3 \\
\hline 5 & AMP-TET-NAL-CFX-SXT- & 1 \\
\hline 5 & AMP-TET-NAL-GEN-SXT- & 1 \\
\hline 5 & AMP-AMS-TET-CHL-SXT- & 3 \\
\hline 5 & AMP-AMS-NAL-CTX-CFZ- & 1 \\
\hline 5 & AMP-NAL-CTX-CFZ-AZM- & 1 \\
\hline 5 & AMP-NAL-CTX-CFZ-SXT- & 1 \\
\hline 5 & AMP-NAL-CFZ-AZM-CIP & 1 \\
\hline 5 & AMP-NAL-SXT-AZM-CIP- & 1 \\
\hline 4 & AMP-AMS-TET-NAL- & 7 \\
\hline 4 & AMP-AMS-NAL-GEN- & 1 \\
\hline 4 & AMP-TET-NAL-GEN- & 3 \\
\hline 4 & AMP-TET-NAL-SXT- & 1 \\
\hline 4 & AMP-TET-CFZ-SXT- & 1 \\
\hline 4 & AMP-NAL-CHL-SXT- & 1 \\
\hline 4 & AMP-NAL-CFZ-AZM- & 1 \\
\hline 3 & AMP-AMS-TET- & 2 \\
\hline 3 & AMP-AMS-NAL- & 1 \\
\hline 3 & AMP-TET-NAL- & 11 \\
\hline 3 & AMP-NAL-CFZ- & 1 \\
\hline 3 & TET-NAL-SXT- & 2 \\
\hline 3 & TET-CHL-SXT- & 3 \\
\hline 2 & AMP-TET- & 4 \\
\hline 2 & AMP-NAL- & 11 \\
\hline 1 & NAL- & 10 \\
\hline
\end{tabular}

\section{Discussion}

\subsection{Definition of Drug Resistance and Multidrug Resistance and the Significance in Detection}

Drug resistance, also known as drug resistance, refered to the resistance of microorganisms to the effects of chemotherapeutic drugs, drug resistance once produced, the role of chemotherapy drugs significantly decreased. Drug resistance was when bacteria lost their sensitivity to the drug, making the treatment ineffective or less effective. Natural pathogens, such as a strain of bacteria, could also had natural resistance. When the antibiotics were used for a long time, most of the sensitive strains were killed, and the drug-resistant strains proliferated to replace the sensitive strains $[10,12-14]$. The significance of our detection was to grasp the drug resistance status and development trend of food-borne pathogens in China, and to provide scientific basis for the prevention of food-borne diseases and rational use of antibiotics in clinic. The results of this surveillance showed that the drug resistance of Salmonella from the faeces of patients with foodborne diseases in our province was very serious. All 123 strains of food-borne Salmonella showed different degrees of resistance to AMP, NAL, TET, SXT, AMS, CFZ, CHL, CTX, CIP, AZM, GEN, CFX, CAZ, but sensitive to IMP and ERY. MIC results were from 1 to $>64(\mu \mathrm{g} / \mathrm{ml})$, and most were distributed in the highest concentration of the drug in the kit.

Multi-drug resistant bacteria which was defined as one microorganism resistanted to 3 or much more categories of antibiotics at the same time, rather than 3 species of the same category of antibiotics. The Kit contains eight categories of antibiotics, 1) $\beta$-lactams: such as AMP, AMS, CFZ, CAZ, CTX, CFX, IPM; 2) aminoglycosides: such as GEN; 3) macrolides: such as ERY, AZM; 4) tetracyclines: such as TET; 5) amido alcohols: such as CHL; 6) quinolones: such as NAL; 7) fluoroquinolones: such as CIP; 8) SULFAs: such as SXT. The results of this test showed that 120 strains of Salmonella were divided into 57 kinds of resistance profile, The most common distribution were AMP-TET-NAL-and AMP-NAL-11 each; the second of all was NAL-10 strains; the third was AMP-AMS-TET-NAL-7 strains. The strains tested were sporadic cases from 22 food-borne disease surveillance sites throughout the province and had no homology. The multi-resistant strains ranged from 3 to 11 kinds of antibiotics, 90 strains were resistant to more than 3 kinds of antibiotics, and the multi-resistant rate was $75.0 \%$. The result was consistent with the current situation in our province. Most patients went to the hospital because the use of antibiotics before the hospital were not better and the patients's diarrhea symptoms were serious, the infected bacteria were resistant to multiple antibiotics. 


\subsection{The Purpose of Serotyping}

At present, there were 2579 serotypings of Salmonella, some Salmonella only infected people; some only infected animals; some infected both human and animals. Except for S. Typhi, paratyphoid A and paratyphoid B which caused human diseases, most of them only infected animals such as domestic animals, rodents and poultry, but sometimes they also caused food-borne diseases by contaminating human food. However, the amount of contamination in food was likely to cause disease [15]. At present, serotyping of food-borne diseases in China can be used as the simplest method of source tracing. By detecting the same serotyping of Salmonella in different samples, we could quickly find out the cause of food, traced the source of infection, recovered the cause of food as soon as possible, and terminated the occurrence of poisoning. The results showed that 123 strains of Salmonella could be divided into 15 serotypings, the main serotypings $\mathrm{S}$. enteritidis accounted for $58.5 \%$, S. typhimurium accounted for $16.3 \%$, and $\mathrm{S}$. stanley accounted for $4.9 \%$. This result was consistent with the strong pathogenicity of various serotypings of Salmonella to human and the high contamination rate in food. Unfortunately, our current system was that the food safety was in charge of the service sector and the Centers for Disease Control and prevention (CDC) were in charge of foodborne diseases. As a result, once a foodborne illness occured, CDC arrived at the scene and it was difficult to collect surplus food. Food-borne diseases can be diagnosed only by collecting samples from poisoned patients, by epidemiological investigation and according to the clinical symptoms of the patients.

\section{Conclusions and hints}

There were different levels of drug resistance and multi-drug resistance in the feces of food-borne patients in Jilin Province, which cause great difficulties in the prevention and treatment of food-borne diseases. In order to prevent and control food-borne diseases, it was necessary to strictly control the adding and using antibiotics in feeding in animal husbandry, aquaculture and other industries. Observed the regulations of drug-rest period and prevented the production and spread of drug-resistant bacteria. The presence of food-borne pathogens and their drug-resistant strains could increase the risk of infection among people with low immunity, such as infants, the elderly and the patients with chronic diseases [16]. Therefore, it was necessary to establish the base data and the database of drug resistance profile, including drug resistance rate, drug resistance profile and drug resistance level of foodborne pathogens in Jilin Province. The significance of drug resistance detection lied in effective and timely warning; people could master accurate epidemiological data; traced the source of drug resistance genes; mastered the transmission path and took timely measures to control infection after discovering drug-resistant bacteria in human and environment. It was very important for the early warning, prevention and treatment of foodborne diseases [17]. Through monitoring the drug resistance of food-borne pathogenic bacteria, human grasped the drug resistance level and the drug resistance trend of the main food-borne pathogenic bacteria in China; preliminary understood the distribution of drug resistance genes of some strains, it also provided basic data for the prediction of drug resistance trend, risk assessment and control strategy, and laid a foundation for the detection of drug resistance genes.

\section{Fund Project}

Health Technology Innovation Project of Jilin Province (2016J034).

\section{References}

[1] Zhang Ling, Liu Guihua. Quantitative detection and Risk Assessment of Salmonella in chicken carcasses [J]. Chinese Journal of microecology, 2015, v 27 (8): 907-909.

[2] Du Xiongwei, Jiang Jie, Li Yan, etc. Detection and analysis of drug resistance of Salmonella Suis [J]. Journal of Dalian Nationalities University, 2017, v 19 (1): 33-35.

[3] Liu Guihua, Guo Changhong, Zhou Fengyan, etc. Analysis of enterococcus contamination and detection results in animal food chain in Jilin Province [J]. China health engineering, 2019, v 18 (6): 815-818.

[4] Liu Guihua, Huang Xin, Zhang Weiyu, Gong Yunwei. Detection and analysis of drug resistance of Salmonella in stool of patients with food-borne diseases [J]. Chinese journal of public health engingeering 2018, v. 17 (01): 25-28.

[5] Li Yueting, Gong Yunwei, Liu Guihua. Analysis of drug resistance of food-borne Salmonella and laxative ESCHERICHIA coli [J]. Chinese Journal of Health Inspection, 2016, v. 26 (22): 3335-3337.

[6] Ministry of Health of the People's Republic of China. GB 4789.4-2016 National Standard for Food Safety Food Microbiology, Salmonella [S]. Beijing: China Standard Publishing, 2017.

[7] Chaudun, Liu Guihua, Qu Li. Analysis of detection results of Salmonella by MIC drug sensitive kit of broth dilution method [J]. Chinese journal of public health engingeering, 2014, v. 13 (4): 314-319.

[8] Li Shunji, Liu Guihua. Detection and analysis of drug resistance of Listeria monocytogene in foods with monocyte proliferation $[\mathrm{J}]$. Chinese Journal of Health Inspection, 2015, v. 25 (20): 3772 -3774.

[9] Li Yueting, Gong Yunwei, Liu Guihua. Analysis of drug resistance of Staphylococcus aureus in Jilin Province in 2014 [J]. Chinese Journal of Health Inspection, 2015, v. 25 (21): 3586-3587.

[10] Zhao Wei, Liu Guihua, Wang Yanqiu, etc. Investigation on contamination and drug resistance of Liszt in food [J]. Chinese Journal of Health Inspection, 2012, v. 22 (6): 1394-1395. 
[11] Li Shunji, Wang Shudong, Cao Tiehong, Yin Dexiu, Chen Shengchen, Liu Guihua. Drug sensitivity test and result analysis of 41 strains of diarrhea-causing ESCHERICHIA coli $[J]$. Chinese journal of public health engingeering, 2016, v. 15 (2): 127-129.

[12] Ren Huijing, Liu Yun. Current status of research on drug resistance of foodborne pathogens [J]. Henan Agriculture, 2013, No. 324 (16): 57+59.

[13] Yang Yang, Fu Ping, Guo Yunchang, etc. Trend of Listeria monocytogene resistance in food-borne mononucleosis in China in 2005 [J]. Health Research, 2008, v. 37 (2): 183-186.

[14] Cui Huixia, Li Wenjie, Zhang Xiuli, etc. SEROTYPING and drug resistance analysis of food-borne Liszt in Henan Province in 2012 [J]. Chinese Journal of Health Inspection, 2014, v. 24 (12): 1800-1803.

[15] Xu Yunmin, Du Yan, Dan Bin, etc.. Surveillance of drug resistance of Salmonella Spp. in CHINET from 2005 to 2014 [J]. Chinese Journal of Infection and chemotherapy, 2016, v. 16 (3): $294-301$.
[16] Wang Fang. Research progress of multi-drug Resistance Gene Island 1 of Salmonella [J]. Chinese Journal of Antibiotics, 2010, v. 35 (06): 414-420.

[17] Zhang Rui, Zhao Xueqin, Shang Xiaochun, etc.. Composition and drug resistance of pathogenic bacteria of infantile diarrhea in Hangzhou [J]. Chinese Journal of Health Inspection, 2015, v. 25 (06): 901-903+905.

\section{Biography}

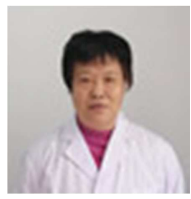

Liu Guihua 1962-, female, Changchun people of Jilin Province, undergraduate, chief physician, Research direction: hygienic microbiology. 